\title{
Public health West Virginia 3.0
}

Danny Scalise II $^{1}$, David Didden ${ }^{2}$

Author Affiliations:

1. Fayette County Health Department, West Virginia

2. Jefferson County Health Department, West Virginia

The authors have no financial disclosures to declare and no conflicts of interest to report.

\section{Corresponding Author:}

Danny Scalise II

Email: danny.f.scalise@wv.gov 


\section{Abstract}

Public health across West Virginia and the nation faces a time of tremendous change. Health is influenced by individual lifestyle, social networks, living and working conditions, and general environmental conditions. As a result, the West Virginia Bureau for Public Health created the West Virginia Public Health Impact Task Force (PHITF) to develop an innovative, custom fit public health system in West Virginia. With goals of improving health, improving quality, and reducing costs, the PHITF recommended a health in all policies approach which combines innovative patient-centered public health services in every community, and asks that local public health partner with stakeholders to align West Virginia's public health system with national recommendations by developing a minimum package of public health services accessible to all West Virginians.

\section{Keywords}

Public Health, Local Health Department

\section{Public health West Virginia 3.0}

Public health departments across West Virginia and the nation face a time of tremendous change, but change is nothing new to this industry. Prior to the American Civil War, public health existed primarily to address epidemic infectious diseases. Around the time of the American Civil War, authorities began developing infrastructure which evolved into state and local health departments. A century later, public health systems adapted to changing conditions by filling gaps in medical care delivery, which eventually became the primary role of local health departments. The challenge of terrorism created a need for public health to change again, by developing responses to this new community health threat. Most recently, public health is being asked to lead our health care system into an era of population health, focusing on value based care delivery models that reward providers for improving entire communities' health and wellbeing.

Life expectancy in the United States has increased tremendously since 1900 and will probably continue to increase for our population as a whole, although subgroups such as middle aged whites are experiencing increased death rates related to substance abuse and suicide. ${ }^{1}$ Public health has helped change a number of the leading causes of death of the last century. However, even with tuberculosis and pneumonia deaths becoming less common, increases in cancer and heart disease deaths per 100,000 have risen dramatically during the same period. ${ }^{2}$

In 2013, West Virginia ranked first with a 27.3\% prevalence for current smoking, first in smokeless tobacco use, ninth in lack of physical activity, and consequently second in fair/poor health status. West Virginia's top rankings also include morbidity indicators such as arthritis, disability, cardiovascular disease, COPD, and diabetes. The state is also a perennial top five in obesity, hypertension, kidney disease, depression, and cancer. To add to the woes, West Virginia ranks in the top ten nationally of mortality indicators including accidents, diabetes, drug overdoses, CLRD, and cancer. ${ }^{3}$

Beyond genetic factors or time in an exam room, health is influenced by individual lifestyle, social networks, living and working conditions, and general environmental conditions. ${ }^{4}$ Clinical 
care accounts for only about $20 \%$ of health outcomes, while social and economic factors impact $40 \%$ of resulting health. Behavioral and environmental variables influence the remainder. ${ }^{5}$ West Virginians' low education rates and higher percentage of population living in poverty certainly are affecting health outcomes. Given the limited influence direct clinical care has on health outcomes, the time has come for public health to engage local physicians in addressing social and behavioral determinants of health.

Health departments in West Virginia have started to assert important roles in improving some of these social determinants of health. Harm reduction programs that reduce transmission of HIV and hepatitis as well as increase access to substance abuse treatment are established in the Cabell-Huntington and Kanawha-Charleston health departments. Other communities are exploring possible partnerships with public health to address the opioid epidemic ravaging our state. Syringe exchange for IV drug users creates an opportunity for public health outreach workers to not only reduce the harm caused by needle sharing, but also exposes clients to learning opportunities about recovery. For example, if an individual suffering from heroin addiction has become homeless, a brief encounter with the syringe exchange nurse could be an opportunity to educate him or her about housing assistance available in the community.

In 2015, under the leadership of the State Health Officer Dr. Rahul Gupta, the West Virginia Bureau for Public Health created the West Virginia Public Health Impact Task Force (PHITF) to develop an innovative, custom fit public health system in West Virginia. ${ }^{6}$ With goals of improving health, improving quality, and reducing costs, the PHITF recommended a health in all policies approach which combines innovative patient-centered public health services in every community, and asks that local public health partner with stakeholders to align West Virginia's public health system with national recommendations by developing a minimum package of public health services accessible to all West Virginians. With strained budgets, public health in West Virginia is currently engaged in conducting an assessment of the current system responsible for the provision of statewide basic public health services including revenue sources with a public policy encouraging the efficient and effective use of public resources for provision of statewide public health services. This includes the increased capacity for local health departments to bill for services traditionally offered at no cost to citizens without health insurance. Another recommendation of the PHITF was to create a Public Health Advisory Board to improve transparency, accountability, and efficiency and promote a statewide culture of health. ${ }^{6}$

The Public Health Accreditation Board reviews application and accredits health departments through a rigorous process designed to protect and improve public health. To achieve accreditation, health departments must document delivery of essential public health services, provide transparency that meets or exceeds public expectations, and demonstrate best practice management processes. ${ }^{7}$ While the idea of health department accreditation is not new, it is only now catching on. As of today, $50 \%$ of the United States population is in a jurisdiction covered by a PHAB Accredited Health Department, though no health department in West Virginia has achieved accreditation. As per the task force's recommendations, local health departments across the state should be working towards becoming accreditation ready. ${ }^{6}$ 
Dwindling state and federal allocations to local health, in the context of expanded health insurance coverage following the Affordable Care Act, create new challenges and new opportunities for health departments. While another safety net provider, the Federally Qualified Health Centers, receives subsidized Medicaid reimbursement on a flat fee per visit basis, health departments cannot bill for many services because nurses provide them. Billing other third-party payers, providing reimbursable disease management and care coordination services, and participating in value-based care delivery arrangements to complement the health care system are potential sources of additional revenue. These solutions will only come to fruition in a wellcoordinated state and local public health system, resiliently embracing adaptation to changing conditions.

\section{References}

1. United States Life Tables, 2011. NVSR Volume 64, Number 11. 63 pp. (PHS) 2015 -1120.

2. Jones D, Podolsky S, Greene J. N Engl J Med 2012; 366:2333-2338.

3. WV Health Statistics Center. (2015). West Virginia Behavioral Risk Factor Surveillance System Report, 2013.

4. Dahlgren G, Whitehead M. Policies and strategies to promote social equity in health. Background document to WHO-Strategy paper for Europe. No. 2007: 14. Institute for Futures Studies, 1991.

5. County health rankings and roadmaps, our approach. [cited $2016 \mathrm{Apr} 1$ ]. Available from: http://www.countyhealthrankings.org/our-approach

6. Final report, West Virginia Public Health Impact Task Force, December 22, 2015, Charleston, West Virginia, U.S.

7. Public Health Accreditation Board. Public Health Accreditation Background. [cited 2016 Apr 1]. Available from: http://www.phaboard.org/about-phab/public-health-accreditation-background 